\title{
¿El surgimiento de un nuevo sujeto de ciudadanía? Aportaciones teóricas al debate contemporáneo sobre los derechos de los niños
}

\author{
Julia Ramiro \\ Carmen Alemán Bracho \\ Universidad Nacional de Educación a Distancia \\ jramiro@der.uned.es; caleman@der.uned.es
}

Recibido: 06-05-2015

Aceptado: 16-10-2015

\section{Resumen}

El artículo discute el concepto de ciudadanía asociado a la infancia. A través de una aproximación analítica, se pretende ofrecer un marco teórico y epistemológico que permita conocer las distintas posturas que sustentan dicho concepto, tanto desde el punto de vista ideológico como filosófico, y que, al mismo tiempo, se encuentran en los diversos debates sobre la naturaleza de los derechos de los niños en las sociedades democráticas. Para ello, se revisan las discusiones contemporáneas en torno al concepto de ciudadanía y se sistematiza y se ordena la literatura científico-social producida, fundamentalmente, a partir de la década de 1980. El artículo concluye con un balance sobre las implicaciones y el impacto de estas corrientes teóricas en la reconceptualización del niño como sujeto de ciudadanía.

Palabras clave: infancia; participación; relaciones de interdependencia; análisis teórico

\begin{abstract}
The emergence of a new subject of citizenship? Theoretical contributions to the contemporary debate on children's rights

This article discusses the concept of children's citizenship. Using an analytical approach, it seeks to establish a theoretical and epistemological framework in order to gain insight into the different perspectives that ideologically and philosophically support this concept and which are, at the same time, involved in the debates on the nature of children's rights in democratic societies. To do so, we review contemporary discussions about the citizenship concept, and systematize and organize the socio-scientific literature produced from the 1980 s onwards. The article concludes with an assessment of the implications and impacts of these theoretical approaches on the re-conceptualization of the child as citizen.
\end{abstract}

Keywords: childhood; participation; interdependency relationships; theoretical analysis 


\section{Sumario}

$\begin{aligned} \text { 1. Introducción } & \text { 4. Implicaciones de los debates sobre } \\ \text { 2. Debates sociopolíticos sobre el } & \text { la ciudadanía e infancia } \\ \text { concepto contemporáneo de ciudadanía } & \text { Referencias bibliográficas }\end{aligned}$

3. Ciudadanía e infancia: corrientes y enfoques

\section{Introducción}

Desde la década de 1990, se asiste a una proliferación de estudios sobre ciudadanía e infancia. El creciente interés científico-social por esta cuestión se debe, en gran parte, a la Convención sobre los Derechos del Niño (CDN), de 1989, primera en inaugurar la figura de los niños y niñas como sujetos legales independientes, al establecer los derechos de participación de estos (artículos 12, 13, 14, 15 y 17), denominados por algunos autores nuevos derechos de ciudadanía (Earls, 2011). No obstante, en esta emergencia, cabe destacar la importancia de los debates desarrollados desde la sociología de la infancia a partir de la década de 1980, pues suponen un claro referente para los estudios, posteriores y coetáneos, sobre ciudadanía e infancia. Principalmente porque, desde la citada disciplina, se asientan las bases para la (re)conceptualización del niño como un sujeto social e individual, con capacidad para participar activamente en la sociedad, en el presente. Punto de inflexión desde el que se incorpora la dimensión política en los estudios sobre la(s) infancia(s) y la vida de los niños y adolescentes: a partir del análisis de las representaciones y los discursos sociales e institucionales (adultos) en torno a la infancia; la posición que, en consecuencia, los niños ocupan en la sociedad (de invisibilidad), y los mecanismos a través de los cuales ellos, como agentes sociales, se oponen, negocian y transforman el deber ser de su estatus como personas menores de edad.

No obstante, si el surgimiento y las aportaciones de la sociología de la infancia, así como la celebración de la Convención sobre los Derechos del Niño de 1989, resultaron la base fundamental de un nuevo interés científico social por el estado de la ciudadanía en la infancia y de los niños, no resultan menos importantes los debates desarrollados sobre el propio concepto de ciudadanía y las críticas planteadas al paradigma clásico, propuesto por T. $\mathrm{H}$. Marshall, desde finales de la década de 1960, con el consecuente surgimiento de los nuevos significados del ser ciudadano. Críticas y planteamientos que abrazan las transformaciones económicas, sociales y políticas acaecidas en las sociedades occidentales a partir de esas fechas, para algunos, democráticas; para otros, (neo)liberales.

Este artículo pretende contribuir al estudio de la ciudadanía y de la infancia, ofreciendo un marco teórico y epistemológico que permita analizar empíricamente la cuestión de los derechos de los niños. Para ello, se realiza una revisión del debate en torno al concepto de ciudadanía y se estudia cómo este ha preparado el terreno para el reconocimiento de los niños como sujetos de derechos. Poste- 
riormente, se proponen y se abordan distintos enfoques que analizan, justifican o reinterpretan la cuestión de la ciudadana en y para la infancia.

Así mismo, el interés de abordar en profundidad los debates académicos desarrollados en torno a la ciudadanía de los niños y de los adolescentes reside en el papel activo que estos desempeñan en la consideración de los niños como ciudadanos. A modo de comunidades epistémicas, esta red de expertos en infancia y en política ha desempeñado un papel fundamental en la discusión y en la consideración de la cuestión de la ciudadanía de los niños. Por un lado, porque algunos participan directamente en organizaciones internacionales a favor de sus derechos, tales como UNICEF, Save the Children o NAT ${ }^{1}, y$, por otro, porque - organizados a través de equipos expertos y de investigaciónejercen una gran influencia en el diseño y en la implementación de políticas, planes y programas para la infancia y la adolescencia.

Por tanto, comprender el recorrido epistemológico y las diversas posturas -ideológicas y semánticas - que conforman los debates en torno a la ciudadanía de los niños supone abordar los contenidos formales y simbólicos que constituyen los marcos que amparan la promoción y/o la denegación del niño como «nuevo» sujeto social y político, es decir, como ciudadano, en las sociedades democráticas occidentales. El artículo concluye con un balance sobre las implicaciones y el impacto de estas corrientes teóricas en la reconceptualización del niño como sujeto de ciudadanía.

\section{Debates sociopolíticos sobre el concepto contemporáneo de ciudadanía}

Desde el paradigma moderno de ciudadanía, la edad y la madurez psicocognitiva y física se erigen como condiciones sine qua non para el acceso y el disfrute de la ciudadanía plena, con lo cual queda en el imaginario social colectivo una fuerte relación entre los conceptos de ciudadano y de adulto ${ }^{2}$.

La relación central de la noción de ciudadanía con el mundo adulto resulta, eminentemente, una herencia histórica que proviene del republicanismo cívico, que fue reelaborada y transformada por la Ilustración en la teoría liberal - a partir del siglo XVIII - y acomodada por el paradigma marshaliano con la creación y la puesta en marcha de los estados de bienestar en la década de 1950.

En el republicanismo cívico, la ciudadanía se adquiría mediante la propiedad, que, a su vez, marcaba la (in)dependencia y a la que se asociaba la razón ${ }^{3}$.

1. Movimiento de Niños Adolescentes Trabajadores.

2. De hecho, algunos estudios, como los de R. Thomson et al. (2004) y R. Lister et al. (2003), muestran la conexión entre la adultez y la ciudadanía en los discursos de los jóvenes. En esta línea de investigación, resultan reveladores los trabajos de J. Hine (2004) y G. Jones y C. Wallace (1992), en los que se muestra la relación existente entre la definición formal de ciudadanía y la identificación con ella — valores democráticos clásicos- por parte de niños y adolescentes, a la luz de los profundos cambios producidos en las democracias y en las sociedades occidentales contemporáneas.

3. Algunos ejemplos históricos que confirman esta tesis pueden ser consultados en la obra sobre el enfoque republicano de A. de Francisco (2007: 121-142). 
Desde este punto de vista, los niños y adolescentes se encontraban en una posición de exclusión en el proceso de formación de la condición ciudadana a través de una variable económico-cultural y no biológica, puesto que la propiedad era y es considerada una posesión adulta ${ }^{4}$ (Roche, 1999: 479).

Ahora bien, el modelo de ciudadanía ha ido cambiando como resultado de las luchas de distintos grupos sociales a lo largo de la historia (Turner, 1993), para, en último término, ser guiado por los principios liberales de igualdad y universalidad. Hasta la década de 1950, la ciudadanía era definida, principalmente, por la posesión de derechos civiles (protegían la propiedad) y políticos (garantizaban la participación en las instituciones políticas).

En el contexto de la construcción y legitimación de los estados de bienestar, T. H. Marshall (1998: 15-74) pretendió resolver el problema de la reconciliación de la estructura formal de la democracia política con las consecuencias sociales del capitalismo como sistema económico. Es decir, trató de reconciliar la igualdad formal con la división de clases, incorporando los derechos sociales al conjunto de derechos que constituían la ciudadanía hasta aquel momento. De esta manera, intentó paliar el impacto negativo de las diferencias de clase en las oportunidades reales de vida, permitiendo, a su vez, cierta inclusión — parcial — a la ciudadanía de grupos que, tradicionalmente, habían sido excluidos de ella (minorías, mujeres, niños y niñas y otros grupos sociales vistos como dependientes). Sin embargo, los derechos sociales por sí mismos tampoco consiguieron erradicar los procesos de exclusión ciudadana de determinados grupos.

En el caso de los niños y adolescentes, se establecía que, por razón de edad, no poseían derechos civiles ni políticos — la condición ciudadana plena se adquiere al cumplir la mayoría de edad-. De hecho, para T. H. Marshall (Marshall, 1998: 34) y el paradigma de ciudadanía moderno (Turner, 1993: 3), los niños son solo ciudadanos potenciales que, a través de sus derechos sociales — fundamentalmente de educación pública—, llegarán a ser adultos y ciudadanos con plenos derechos y obligaciones. La minoría de edad designa, pues, la condición de dependencia de los niños y adolescentes en referencia a sus familias o a las instituciones públicas que los atienden, ya que, por alegadas razones biológicas, no han adquirido las herramientas suficientes para ostentar un estatus pleno de ciudadanía, necesitan de una protección especial y se enfatizan en ese momento los derechos de bienestar de la infancia (salud, familia y educación). De hecho, para H. Wintersberger (2006: 90-91), el estado de bienestar se presenta principalmente como un sistema adultista y paternalista, porque las políticas sociales de infancia se encuentran entre las clases de políticas públicas de menor interés o, en el caso de ser tenidas en cuenta, se llevan a cabo de forma paternalista, siendo los adultos quienes deciden qué es lo más adecuado para el niño («mejor interés del menor»), con lo que prevalece, por

4. P. Ariès (1962) señaló que hasta el siglo Xviır no existieron vocablos para designar a los niños en el sentido actual. Niño, muchacho e infante se utilizaban indistintamente para hacer referencia a las personas que no tenían posesiones y que, por tanto, dependían de otras. 
tanto, el familismo en la actitud frente a este. Como consecuencia, L. ShamgarHadelman (1994) sugirió que los niños se encuentran invisibilizados y silenciados por la ideología de la familización, pues, incluso cuando estos son incluidos en los programas públicos, lo son en términos, principalmente, de protección.

Construir la infancia exclusivamente en torno a la edad supuso la fetichización de la inocencia del niño, cuyo coste fundamental fue la denegación de su capacidad política y social, así como de su ciudadanía. Esta representación colectiva, fundamentada en las ciencias del desarrollo del niño, quedó articulada a través de la ideología del proteccionismo y en la noción del niño como ciudadano del futuro. Cuestión que, si bien a lo largo del siglo xx fue tomando cada vez más fuerza, fue contestada, casi por primera vez, por el Movimiento para la Liberación de los Niños (Children's Liberations Movement, CML), en la década de $1970^{5}$, que reclamaba los derechos políticos para el conjunto de la infancia, criticando duramente la práctica tradicional de su protección.

No obstante, es a partir de la CDN de 1989 cuando se produce un cambio sustantivo respecto del lugar político (simbólico) que ocupa el niño en la sociedad. El interés científico y social comienza a abordar la condición ciudadana de los niños, abriéndose un debate en el que se pone de manifiesto la centralidad de la idea de participación y la agencia de los niños y adolescentes en el proceso de adquisición y disfrute de su ciudadanía, sin duda precedida por los debates internos sobre la naturaleza de la misma y desarrollados a partir de las críticas al paradigma clásico de T. H. Marshall. Debates que se asocian, por un lado, a los modelos de la diferencia y a los movimientos postcolonialistas, surgidos en la década de 1960, y, por otro, a la acción gubernamental de la «nueva derecha» (Reagan en EE. UU. y Thatcher en Gran Bretaña ${ }^{6}$ ), desarrollada a partir de la década de 1980. De esta manera, como han señalado J. Benedicto y M. L. Morán (2002: 45), para la reflexión sociopolítica, las ciencias sociales incorporan cuestiones como la naturaleza de la democracia, el concepto de representación, el vínculo entre libertad e igualdad, la concepción de justicia, etc.

Es así como los profundos cambios sociales, económicos y políticos acaecidos a partir de la década de 1980 precipitaron la necesidad de revisar el propio concepto de ciudadanía hacia un modelo más inclusivo, el cual permitiera clarificar y ordenar las relaciones entre los (nuevos) sujetos sociales y las instituciones, también democráticas.

5. M. Liebel (2006b) señala que, desde el principio del siglo xx, en algunos países, comenzaron a surgir movimientos que luchaban por los derechos de autonomía y de participación política de los niños. Como resultado de ese proceso, destaca la Declaración de Moscú sobre los Derechos del Niño y la Niña (1918), así como la actividad y las obras del pediatra y pedagogo polaco J. Korczak, quien, como director de un orfanato, promocionó el rol independiente y activo de los niños que estaban a su cargo, luchando por la extensión de los derechos y por la igualdad de estos niños en la sociedad.

6. Las críticas planteadas al paradigma clásico de ciudadanía y al estado de bienestar por la «nueva derecha», abanderada por M. Tatcher y R. Reagan, se dirigieron, principalmente, a «denunciar» que los ciudadanos se han convertido en sujetos pasivos, a partir de los servicios y las prestaciones del estado de bienestar (derechos sociales), y en clientes dependientes de las instituciones públicas. 
La idea de la ciudadanía asociada a la infancia se incorporó institucionalmente, en gran parte de los países occidentales, en los términos de los cambios políticos llevados a cabo por «la nueva derecha» en la década de 1980, definidos, en parte, por la promoción de la naturaleza activa de la ciudadanía en términos de sus responsabilidades, sus obligaciones y su implicación ${ }^{7}$ (Hackett, 1997: 82-83).

A partir de estas fechas, y a través de la crítica neoliberal a los estados de bienestar, los derechos sociales ligados a la infancia son devaluados ${ }^{8}$. Se desarrolla un discurso institucional y político que apela a la ciudadanía en el sentido activo, es decir, hacia la responsabilidad. Y este discurso permea en la cuestión de la infancia, donde la idea de la ciudadanía activa se convierte en un elemento central, donde la participación infantil y juvenil se presenta como un asunto fundamental para la supervivencia de las instituciones y de los sistemas democráticos tradicionales.

En este sentido, tanto la idea de ciudadanía activa — cuya matriz la constituyen los derechos de participación-como la incorporación de los nuevos «derechos de cuarta generación» — asociados a una idea de justicia global, entre los que destacan los derechos ecológicos y los culturales-, tienen que convivir y encontrar el equilibrio con la definición clásica de la ciudadanía, fundamentada en la titularidad de derechos. Así surgen las nociones de ciudadanía activa y de ciudadanía global frente al paradigma clásico, las cuales incorporan dos grandes tensiones propias de finales del siglo xx y de comienzos del siglo XXI. Por un lado, la clásica dialéctica entre la pasividad de los ciudadanos y la participación de los mismos en la construcción de los espacios públicos y, por otro, las relaciones complejas en la atribución de la capacidad y las competencias

7. Para la teoría liberal clásica, la ciudadanía como práctica designa la relación del individuo con la comunidad política, lo cual genera un marco para la interacción entre los individuos dentro de la sociedad civil (Faulks, 2003: 107). En este sentido, la puesta en escena de la idea de ciudadanía activa apela a la implicación y a la responsabilidad de los ciudadanos para con su comunidad política, en términos de instituciones democráticas clásicas y representativas. De hecho, las responsabilidades y las obligaciones ciudadanas son vistas como el nexo de unión entre la ciudadanía como estatus y la ciudadanía como práctica. Por tanto, desde esta línea de pensamiento, la ciudadanía de la infancia se teoriza sobre el discurso del entrenamiento político, democrático y ciudadano. Es decir, en una versión institucionalizada de la ciudadanía, se trata de generar ciudadanos desde «arriba» que, desde su infancia, se encuentren familiarizados con los valores cívicos y democráticos establecidos, a través de una implicación participativa de estos en una suerte de órganos consultivos especialmente diseñados para ellos.

8. Para Marshall (1998), los derechos (especialmente los derechos sociales) son la fuerza integradora de los individuos a su pertenencia (participación) ciudadana. Por el contrario, para pensadores como J. M. Barbalet (1988), la ciudadanía es el derecho a la participación en la política. Así, los derechos sociales facilitan dicha participación, más que constituir una parte de esa ciudadanía. Por otra parte, el autor pone de manifiesto la tensión existente entre la «ética de la participación» y la naturaleza pasiva de los derechos sociales, constitutivos de los estados de bienestar, en tanto que, para J. M. Barbalet (1988), los derechos sociales a menudo estigmatizan más que empoderan a sus titulares para ejercer la ciudadanía, en términos estrictamente políticos. 
políticas entre los estados nación y el orden internacional, como instituciones legitimadoras, generadoras y protectoras de derechos y ciudadanía. De ahí las tensiones existentes entre distintas líneas de pensamiento en torno a la ciudadanía y, en consecuencia, sobre su aplicación, ampliación y/o incorporación de los niños y niñas.

En la medida en que la globalización y los derechos humanos están posibilitando un nuevo marco de reivindicaciones, se constituyen como elementos de un nuevo discurso sobre los derechos de la ciudadanía. «El terreno analítico, en el que debemos situar la cuestión de los derechos, de la autoridad y de los deberes, está efectivamente experimentando grandes transformaciones» (Sassen, 2003: 99). En este contexto, algunos autores han apelado a la idea del niño como ciudadano global (Stasiulis, 2002; Van Bueren, 2011).

Esta postura - ligada a la idea de ciudadanía activa y apoyada en la labor de organizaciones internacionales como UNICEF o Save the Children-ve la CDN de 1989 como una declaración de universalidad de los derechos de los niños y adolescentes ${ }^{9}$ y establece el énfasis en el conjunto de derechos de participación contenidos en la misma (artículos 12-17). No obstante, como sugiere D. Stasiulis (2002), si bien la CDN de 1989 ha promovido un modelo de ciudadanía activa —y global — para los niños, dicho modelo sigue siendo difícilmente reconciliable con la idea occidental de infancia, aun dominante, que fetichiza la inocencia, la pasividad y la incompetencia de los niños ${ }^{10}$.

Así, frente al entusiasmo académico y político por la CDN de 1989 y por la idea de ciudadanía global, formulada para incorporar a los niños y adolescentes como sujetos plenos de ciudadanía, algunos autores como N. Lee (2005) plantean que, incluso a principios del siglo Xxi, la noción del niño dependiente sigue formando parte del desarrollo histórico de las políticas nacionales sobre infancia y que la CDN de 1989 resulta un instrumento regulador y de control internacional ante el declive del poder de los estados.

\section{Ciudadanía e infancia: corrientes y enfoques}

A pesar de la extensión y de la profusión de los discursos sobre la ciudadanía de los niños, definir este concepto se encuentra, a día de hoy, lejos de ser una tarea fácil. Los discursos y las teorías sobre la ciudadanía de los niños y de los adolescentes incluyen numerosos conceptos: derechos y obligaciones, igualdad y diferencia, estatus y práctica, pertenencia, dignidad, competen-

9. Este enfoque, en su vertiente más filosófica, establece que la condición de ciudadanía y los derechos humanos mantienen una relación simbiótica (Van Bueren, 2011:31).

10. En contraste con la incapacidad de los estados para incorporar la muy teorizada pero poco practicada ciudadanía activa de los niños, D. Stasiulis (2002) propone un modelo alternativo emanado de las movilizaciones protagonizadas por el movimiento Free The Children, que, para la autora, constituye un ejemplo de los niños como actores sociales, capaces de organizarse y participar activa y políticamente en el mundo y sobre las cuestiones que les afectan. 
cia, autonomía, dependencia e independencia, participación y agencia. La definición e inclusión o no de dichas nociones son las que establecen la existencia de distintas posturas analíticas y discursivas, que tratan de responder, en último término, a la pregunta planteada por M. Hill y K. Tisdall sobre si los conceptos de infancia y ciudadanía son compatibles (1997: 38), lo cual dependerá de cómo es definida la ciudadanía y cómo lo es, al respecto, la infancia, y, al mismo tiempo, sobre si la primera permite la inclusión de la segunda o no.

Para facilitar el acceso a las posturas teórico-filosóficas existentes, se plantea una estructura basada en la proposición de dos corrientes principalmente asociadas a cómo es entendida la idea de ciudadanía: eminentemente, como titularidad de derechos (ciudadanía legal) o como práctica sociopolítica (ciudadanía sustantiva). Dentro de dichas corrientes, se propone otra subcategorización que, en la figura de los enfoques, ordena y sistematiza los significados en torno a la infancia y sus atributos y de cómo estos se relacionan con la mencionada ciudadanía.

\subsection{Corriente legal}

La corriente legal entiende la ciudadanía como un estatus de derechos (civiles, políticos y sociales) y responsabilidades cívicas que el individuo posee por pertenecer a un estado nación y que adquiere, plenamente, cuando alcanza la condición adulta y la madurez suficiente para hacer uso de sus derechos. Por tanto, esta corriente centra su interés en la condición delegada de la ciudadanía de los niños, ya que estos, en la actualidad, son sujetos ciudadanos a través de su vinculación al Estado y a la familia, no como sujetos independientes, a pesar de lo establecido por la CDN de 1989.

No obstante, según la clasificación de M. Moosa-Mitha (2005: 369-388), esta corriente se compone de tres enfoques que teorizan los derechos de ciudadanía de los niños y niñas de manera diferente: Child Liberationist, paternalismo y proteccionismo liberal. Los tres articulan sus estudios a partir de la oposición de las categorías formadas por niño y adulto e igualdad y diferencia. Mientras que el paternalismo liberal y el proteccionismo han enfatizado las diferencias - en términos de razón y posesión de derechos- existentes entre niños y adultos, el Child Liberationist va a reclamar una relación de igualdad entre ellos, en tanto que considera la «línea» divisoria entre niños y adultos como una cuestión totalmente cultural y eminentemente arbitraria (Franklin, 1986b: 7 y 11).

\subsubsection{Child Liberationist}

Sin duda, Child Liberationist es el enfoque más radical y el que menos literatura ha producido en los últimos veinte años. De esta manera, se plantearán las dos ideas claves que articulan sus propuestas para centrar la discusión entre el paternalismo liberal y el proteccionismo, siendo este último el enfoque que domina en los estudios sobre ciudadanía de la infancia. 
En la década de 1970, surge el movimiento Child Liberationist, cuyos máximos exponentes son R. Farson (1974) y J. Holt (1975), que reclaman una posición de plena igualdad entre niños y adultos (Holt, 1975: 15).

Para el Child Liberationist, existe una necesidad perentoria de reconocer los derechos civiles y políticos de los niños, pues, mientras les son negados, se perpetúa el sistema de dominación sobre ellos en el contexto de las democracias occidentales (Cohen, 1980; Hougalte, 1980). Para este enfoque, ni «el mejor interés del menor", ni las políticas proteccionistas son capaces de dar cobertura a las necesidades reales de los niños y niñas. De hecho, este enfoque va a exponer que la idea de «niño» se ha construido y reconstruido a lo largo de la historia para responder a los intereses del mundo adulto. Así, la lógica paternalista se presenta como una forma de legitimar la dominación de los adultos sobre los niños, por lo que las únicas vías de liberación de la infancia como minoría oprimida serán la autodeterminación (Farson, 1974: 27; Holt, 1975: 15) y la formación de grupos de liberación, integrados por los propios niños en la reclamación de sus derechos.

Recientemente, en la línea del debate sobre los derechos políticos de los niños, D. Archard (2004: 98) y P. Kirbi y M. Woodhead (2003: 268-270) señalaron que la negación del derecho al voto es una condición ineludible de la exclusión de la ciudadanía.

Aunque la CDN de 1989 establece y reconoce algunos derechos políticos a los niños (artículo 12-17) y, por tanto, esta ha sido vista como el inicio en el reconocimiento del niño ciudadano, para algunos autores, el dilema central que continúa excluyendo a las personas menores de edad de los derechos políticos es la negación del voto para los niños. Es decir, en un contexto político representativo, el voto se propone como uno de los mecanismos fundamentales de participación política en el sistema democrático. Por tanto, mientras los niños no tengan reconocido tal derecho, no llegarán a constituirse como ciudadanos plenos y permanecerán marginados de muchos de los aspectos de la participación política.

Según dicho enfoque, la marginación ciudadana de los niños encuentra su razón de ser en la representación de estos en términos de incapacidad para ejercer sus derechos y para entender las cuestiones políticas, situación que, institucionalmente, cambia cuando llegan a la mayoría de edad (establecida en los 18 años en gran parte de los países occidentales).

Sin embargo, al mismo tiempo, han señalado que, en muchos países, la responsabilidad penal del niño ante sus actos delictivos es establecida por las leyes por debajo de la mayoría de edad ${ }^{11}$, lo cual desvela las ambivalencias subyacentes al establecimiento de la capacidad de los niños de razonar o de ser conscientes de sus actos asociados con la edad biológica (Archard, 2004: 132-133), cuando ellos son establecidos como el primer objetivo del comportamiento antisocial.

11. Por ejemplo, en Gales e Inglaterra, actualmente, está fijado en los 10 años (Kirbi y Woodhead, 2003: 269), y en España, en los 14, según la Ley 5/2000 de la responsabilidad penal del menor. 
Al respecto, E. Such y R. Walker (2005) pusieron de manifiesto la contradicción existente entre la atribución de responsabilidades a los niños en materia penal y de «comportamiento antisocial» y el lugar que ocupan en las leyes sobre familia. Es así como, para los autores, parece que los niños son capaces de ser deliberadamente irresponsables pero no deliberadamente responsables (Such y Walker, 2005: 46).

En consecuencia, Child Liberationist plantea que la condición de madurez responsabilidad y capacidad de los niños y adolescentes no responde tanto a la edad como al estatus social que las instituciones y los gobiernos les permiten y les adjudican. Y es así como, desde sus inicios, este enfoque revindicó lo indispensable de incorporar a la infancia en los procesos políticos y de toma de decisiones democráticos, así como la ampliación de los derechos de la misma hasta alcanzar la plena igualdad con los adultos ${ }^{12}$ (Cohen, 1980), pues, a pesar del establecimiento de la CDN de 1989 y de las consiguientes leyes nacionales desarrolladas al amparo de esta, los derechos de los niños y niñas continúan siendo una segunda clase de derechos, no comparable con la de los adultos.

Si tanto los derechos políticos, en un sentido fuerte, como los civiles no han sido reconocidos plenamente para los niños, por considerarse que son inapropiados e irrelevantes para la infancia (Cohen, 2005: 224), tampoco los derechos sociales parecen pertenecer plenamente a los niños, pues son derechos de ciudadanía social de los que ellos se benefician por "proximidad» a sus padres.

\subsubsection{Paternalismo liberal y proteccionismo}

El paternalismo liberal y el proteccionismo parten de asunciones semejantes sobre los niños y su ciudadanía, y se diferencian en la distribución de las responsabilidades entre familia y Estado, respectivamente. De esta manera, el debate entre ellos no se ha centrado tanto en la condición de semiciudadanía de los niños y niñas, ampliamente aceptada por ambos, como en el papel de la familia y de las instituciones públicas a la hora de guiar y proveer de derechos a estos; en especial, aquellos relacionados con la educación para la futura ciudadanía. El debate entre estos dos enfoques es, en el fondo, el debate entre el liberalismo y el proteccionismo, entre los principios de libertad y de igualdad.

Siguiendo la tesis de T. H. Marshall (1998: 34), ambos enfoques entienden que el niño se encuentra en una etapa de formación y de adquisición de las capacidades y de la madurez necesarias para ser futuros ciudadanos. Así, los niños y niñas no obtienen los derechos de ciudadanía por sí mismos, sino que acceden a dicho estatus por la pertenencia a un estado nación y a su familia. Es decir, bien por el derecho de sangre - ser hijos de padres que ostentan la condición ciudadana - o bien por el derecho de suelo - haber nacido dentro del territorio de un país— (Ben-Arieh y Boyer, 2005: 36; Mandel, 1995: 265-280;

12. Entre estos derechos, destacan el derecho a la información y el derecho al voto político (Farson, 1974: 175-180). 
Shachar, 2003: 345) ${ }^{13}$. La razón de este proceso, establecen, se debe a que el niño es aún un sujeto dependiente y vulnerable que necesita de la guía adulta (O’Neill, 1988: 461). Aun así, el niño o adolescente, dependiendo de sus circunstancias y de la naturaleza de la situación que le afecta, será capaz de participar en los procesos en los que se ve inmerso (Lansdown, 2003: 280).

El paternalismo liberal considera que el niño pertenece, eminentemente, al ámbito de lo privado y establece el énfasis de su ciudadanía en el papel educativo - y la protección- de las familias. La familia es la que tiene la obligación y la responsabilidad de educar —a través del principio del «mejor interés del niño» (Goldstein et al., 1979) — a sus hijos, en los mejores valores y preceptos éticos, para que, en un futuro, sean ciudadanos integrados en su sociedad, pues el interés de los padres incorpora por definición el interés de sus hijos (Galston, 2003: 211- 233). En este sentido, el Estado debe preservar los derechos de libertad de las familias, siendo únicamente intervenidas cuando, en el ejercicio de tal libertad, se vulneran los derechos de los niños o se amenaza la constitución del orden público.

De hecho, S. Burtt (2003: 266) refuerza esta tesis cuando plantea que la autoridad paterna es una condición que se justifica a la luz del bienestar de la infancia y que esta finalidad es la que impide que se ejerzan prácticas parentales de abuso y de vulneración de los derechos de la misma. En consecuencia, «el mejor interés del niño» viene a designar una serie de prácticas dirigidas específicamente a sus necesidades y no a su incapacidad para razonar. Para la autora, el hecho de definir la autoridad paternal a partir de la falta de razón de los niños conlleva el riesgo de ser demasiado proteccionistas, pues al Estado le interesa incidir en la manera y en los valores en que se forma a los futuros ciudadanos (Galston, 2003). Punto del debate que remite, directamente, a la adecuación, o no, y a considerar hasta qué punto el Estado debe ampliar los derechos de los niños, particularmente de ciudadanía, tras la ratificación de la CDN de 1989. Para O. O’Neill (1992), la respuesta es negativa, pues se expone a los niños a una situación para la que no están preparados. La teoría liberal de la ciudadanía plantea que, para obtener derechos, hay que ser «capaz» de asumir iguales responsabilidades. Así, la autora establece que «existen buenas razones para no basar nuestros argumentos en el llamamiento de los derechos fundamentales de los niños [...] su remedio fundamental en madurar» (O’Neill, 1992: 39).

El enfoque proteccionista surgió para mitigar los efectos de desigualdad que pueden resultar de la condición delegada de los niños y de su posición como minoría de poder en la sociedad. De esta manera, se propone avanzar en los derechos de ciudadanía de los niños profundizando en sus derechos sociales

13. En general, la mayor parte de los países suelen combinar estos dos tipos legales de adquisición de la ciudadanía, dando prioridad a una de ellas en función del tipo de sociedad de que se trate. Si la identidad y la condición ciudadana se vincula estrechamente con la identidad nacional, predomina la fórmula del derecho de sangre, como en el caso de España. Por el contrario, en sociedades multiculturales y plurales, como en el Reino Unido, se suele dar preferencia al derecho de suelo, a través de la fórmula, en muchos casos, del arraigo. 
a través del establecimiento de sus derechos de protección. De ahí que, a la inversa que los adultos, en la jerarquía de los derechos de los niños y niñas, se encuentren los derechos sociales, siendo progresivamente establecidos, de acuerdo con la edad, los derechos civiles y, por último, los derechos políticos. De esta manera, se apela a la responsabilidad de los estados en el establecimiento y en la protección de los derechos de ciudadanía de los niños, pues confiar tan solo en que las familias van a representar su «mejor interés» es arriesgado (Ben-Arieh y Boyer, 2005: 47-48; Fineman, 2003: 234; Knutsson, 1997: 140).

Desde este enfoque, el reconocimiento institucional de los derechos de ciudadanía de los niños y adolescentes es muy importante, pues la definición y la forma de tal estatus (qué derechos comporta) es la que va a determinar el bienestar de los ciudadanos en sentido material (es decir, provisión de servicios y defensa de sus intereses) y la que va a promocionar un bienestar psicológico asociado a la identidad ciudadana. A. Ben-Arieh y Y. Boyer (2005: 34), a través del caso israelí, establecen que el tipo de ciudadanía que ostentan los niños es esencial para su desarrollo y bienestar, principalmente, porque este es un periodo en la vida de las personas caracterizado por unas necesidades específicas, en su mayoría de protección, y porque en esta etapa se forjan las identidades adultas — una identidad ciudadana — relacionadas con la posición y el reconocimiento que obtienen los niños en su comunidad. M. Freeman (1992a) sostiene que una persona a la que no se le reconocen los derechos de ciudadanía se encuentra en una posición análoga a la del esclavo, es decir, se encuentra a disposición de otros. De ahí que plantee la importancia de favorecer la condición ciudadana de los niños como única vía para procurarles un mínimo de bienestar y compensar su posición de subordinación, garantizándoles una serie de derechos, entre los más importantes, los de protección y de participación (Roberts, 2001: 61).

El proteccionismo considera relevantes los derechos de participación de los niños establecidos por la Convención de 1989 (derecho a ser escuchados y a tener en consideración su punto de vista), atendiendo a que estos no deben menoscabar sus derechos de protección y que, siempre, deben ejercerse en el marco de las instituciones familiares y estatales (principalmente, las educativas), lo cual permite guiar y autorizar el desarrollo de la participación y el aprendizaje del sentido de la ciudadanía. De hecho, sostiene que la participación (ciudadana) de los niños encuentra el mejor espacio de práctica en las familias y en las escuelas, ambas, a su juicio, instituciones orientadas a garantizar el bienestar de la infancia. La idea que aquí se defiende, y que la diferencia del paternalismo liberal, es que los niños han de ser visibles y tomados en cuenta, tanto en el ámbito privado de la familia como en el espacio público y político del Estado, es decir, la obligación de garantizar los derechos de los niños debe ser compartida para, así, evitar que se vulneren los derechos de estos como consecuencia de su invisibilidad en lo privado, por lo que el proteccionismo da un paso más allá respecto al paternalismo liberal, al encarecer el ejercicio de la ciudadanía de los niños en forma de participación activa en su entorno, a través de la relación con los que les rodean y/o mediante estructuras (in)formales de 
participación (movimientos juveniles, etc.), dirigidas a su representación en la comunidad. Así, para el enfoque proteccionista, la percepción adulta resulta esencial en el reconocimiento del niño ciudadano, aunque matiza al señalar la necesidad de incorporar en él la visión de los niños (Knutsson, 1997: 149).

\subsection{Corriente sustantiva}

Esta corriente parte de una definición de ciudadanía más amplia e inclusiva que la anterior — ciudadanía pasiva_- pues, más allá de la posesión de un estatus de derechos, la ciudadanía, para ella, es el ejercicio, como sujetos política y socialmente activos, de tales derechos.

J. Benedicto, redefine el concepto de ciudadanía en función de tres objetivos fundamentales:

1. Construir una noción dinámica de ciudadanía en la que el centro de interés sea su dimensión relacional y las prácticas sociales.

2. Reconocer el carácter multidimensional del concepto de ciudadanía, permitiendo analizar su dinámica social en profundidad.

3. Recuperar la centralidad sociopolítica del concepto cuando se discute sobre ciudadanía y ciudadanos.

Según el autor, estos planteamientos permitirían avanzar en el conocimiento de dos procesos fundamentales, por un lado, cómo funciona y cómo se transforma la ciudadanía y, por otro, cómo se llega a ser ciudadano (Benedicto, 2007: 2).

De esta manera, esta corriente intenta desplazar el debate de la dependencia de los niños hacia el de la participación, es decir, cómo y a través de qué mecanismos - personales e institucionales - los niños y niñas son capaces de actuar en el mundo que les rodea, posibilitando, así, su consideración como ciudadanos del presente (Jans, 2004: 40).

En este sentido, el centro de interés de esta corriente no es tanto la condición de semiciudadanía de los niños, ni el énfasis en su titularidad de derechos, como la capacidad de estos para participar en los procesos sociales, culturales, económicos y políticos que les afectan.

No obstante, dentro de esta corriente, se pueden hallar dos enfoque distintos y complementarios: ciudadanía diferenciada (differentiated citizenship) y ciudadanía vivida (lived citizenship) ${ }^{14}$. Ambos enfoques parten de una conceptualización similar del niño, la posición que ocupa en la sociedad y las desigualdades sociales y simbólicas que le afectan como tal, por lo que ambos enfoques comparten la crítica fundamental al modelo de ciudadanía dominante, que justifica la exclusión social, económica, cultural y política de la infancia. La

14. Clasificación tomada de A. James (2011) en «To be (come) or not to be (come): Understanding children's citizenship» y esbozada, previamente, por M. Liebel (2008) en "Citizenship from below». 
diferencia entre ellos reside en la formulación de propuestas teóricas sugeridas para modificar y reelaborar esta situación. Mientras que, para el enfoque de la ciudadanía diferenciada, la revisión del propio concepto de ciudadanía es una cuestión prioritaria; para el enfoque de la ciudadanía vivida, la centralidad recae en la idea de agencia o de la inclusión de los niños en el espacio público y privilegiado de los derechos a través del reconocimiento de su capacidad para participar activamente en la sociedad.

\subsubsection{De la diferencia a la igualdad diferenciada (differentiated citizenship)}

El enfoque de la ciudadanía diferenciada tiene como referente a los modelos de la diferencia, en la medida en que, a partir de la década de 1960, los «nuevos movimientos sociales e identitarios - tales como el postcolonialismo, el antirracismo, el feminismo, los movimientos de gays y lesbianas, etc.- fueron los encargados de trasladar a la arena pública la condición de marginalidad, exclusión y, en el mejor de los casos, de semiciudadanía de determinados grupos sociales por razón de su "diferencia» (clase, género, etnia, religión, etc.), respecto del paradigma dominante que definía al ciudadano tipo.

$\mathrm{Al}$ igual que para los modelos de la diferencia, este enfoque plantea que la ciudadanía plena requiere de, obligatoriamente, una condición de reconocimiento público y político de las diferencias, de los intereses y de las necesidades de los distintos grupos sociales que constituyen la sociedad. En este sentido, la llegada de la infancia, como cuestión social relacionada con la ciudadanía, es analizada como el siguiente paso, obvio e inevitable, en el establecimiento de los procesos de fragmentación dentro de los grupos identitarios (King, 2007: 203).

Es importante tener en cuenta que los estudios, insertados en este enfoque, basarán gran parte de su armazón teórica en los modelos de la diferencia - particularmente en la crítica feminista-, en cuanto que estos desafiaron los principios liberales de ciudadanía, desde los cuales los niños y adolescentes se ven excluidos, lo cual generaría nuevas formas de entender la ciudadanía para hacerla inclusiva a ellos.

El paradigma liberal de ciudadanía ubica a las identidades de grupo y a sus diferencias en el ámbito de lo privado, es decir, en términos de libertades negativas garantizadas por la ley y los derechos de acuerdo con el principio de igualdad (Rawls, 1971), lo que, en términos de las teorías de la diferencia, justifica la exclusión "democrática» de determinados grupos de los espacios de la ciudadanía. De ahí que los modelos de la diferencia, así como el enfoque de la ciudadanía diferenciada, basen gran parte de su análisis en la dialéctica entre libertad e igualdad y ámbito público y ámbito privado contenida en el modelo de justicia liberal y, por ende, de ciudadanía.

La ciudadanía liberal se construye a partir de los principios de libertad, igualdad y universalidad, donde las diferencias identitarias no son incorporadas, en la medida en que, para este paradigma, pertenecen al ámbito de la vida privada de las personas. El espacio de la ciudadanía es, por tanto, un espacio neutro, independiente e imparcial ante las identidades de grupo o de las condiciones de existencia de los sujetos (Rawls, 1971: 190). 
El paradigma liberal de ciudadanía entiende la igualdad en términos de igualdad de oportunidades ${ }^{15}$, es decir, sostiene que todos los miembros de la sociedad (individuos) poseen los mismos derechos y, por tanto, son tratados con la misma dignidad e idéntico respeto ante la ley (neutralidad). Por su parte, el principio de libertad corresponde a la idea de libertad negativa: la falta de intervención de lo político (el Estado) en los intereses de los ciudadanos (los individuos), donde la ley y los derechos se erigen como una barrera protectora para salvaguardar la privacidad de cada individuo y sus intereses, con lo cual se evitan las interferencias entre las actividades y las decisiones de unos ciudadanos y otros.

En consecuencia, los ciudadanos son iguales poseedores de derechos y tienen la libertad para participar, o no, en sus comunidades políticas en virtud de su razón (madurez y capacidad) y su autonomía (independencia), elementos centrales en la construcción moderna de la ciudadanía.

Así, la ciudadanía es entendida por este paradigma, fundamentalmente, como un estatus de derechos pasivo, y la participación, como toma de decisiones individuales racionales y autónomas. Esto quiere decir que aquellos que no posean dichas capacidades verán representados sus intereses a través de la participación de otros, preservando, así, sus derechos y, por ende, su posición de igualdad y libertad en la sociedad.

Los modelos de la diferencia se construyeron en oposición al paradigma liberal a través de las críticas planteadas a sus conceptos de libertad e igualdad, en la medida en que estos se fundamentan sobre la idea de una razón universal, justificando el sometimiento de la diferencia al poder de ciertos grupos y a la exclusión o marginación de determinados sujetos de la definición de ciudadanía (Young, 2000: 171-191).

Así, para los modelos de la diferencia, la libertad no es una libertad negativa (privacidad), sino el derecho a participar, desde las diferencias, en las instituciones políticas y sociales, donde la igualdad es el derecho a ser ciudadanos «diferentemente iguales», reconocidos y con posibilidad de incidir en la vida pública (Young, 2000: 201-203).

La participación en la sociedad, por tanto, es una condición sine qua non de la ciudadanía, que pasa necesariamente por el reconocimiento público de la diferencia, por lo cual rompe con el principio de neutralidad (universalidad), pues si la ciudadanía no se construye teniendo en cuenta las diferencias y las desigualdades que existen en la sociedad, difícilmente podrá aspirar a la igualdad y, menos, a la universalidad. Por tanto, las diferencias no son parte de la vida privada de las personas, sino una cuestión pública y política que, mientras no sean reconocidas, seguirán siendo objeto de opresión y de exclusión del espacio cívico (Young, 2000: 197).

El reconocimiento de tales diferencias implica, pues, la inclusión sociopolítica y la participación de los distintos miembros de la sociedad, a pesar

15. B. Siim plantea que la igualdad en el modelo liberal es entendida más como «igualdad de oportunidades» que como un hecho y como estatus más que en términos estructurales (Siim, 2000). 
de que no coincidan con la imagen de sujeto, cuyas virtudes son la razón y la independencia, asociadas a determinados grupos (tradicionalmente, el varón blanco propietario) y no a otros. Es así como las mujeres, durante mucho tiempo, fueron excluidas de dicha definición a través de las representaciones de grupo como «dependientes» $\mathrm{y}$ «emocionales»; los grupos raciales, por su «incapacidad para razonar»; etc.

Para M. Moosa-Mitha (2005: 371 y 374), mientras que estos grupos han venido consiguiendo la inclusión ciudadana, al menos en el aspecto formal, los niños siguen siendo considerados «aún no ciudadanos» debido a esta definición de ciudadanía, atravesada por criterios de racionalidad, de independencia y de autonomía.

De estas consideraciones, se desprende que, ante el paradigma liberal de ciudadanía, los modelos de la diferencia hacen especial hincapié en el sentido sustantivo (Marshal, 1998) o activo (Turner, 1993: 3) de la ciudadanía, planteando, necesariamente, una redefinición de los espacios públicos y privados, porque, para ellos, la participación y la presencia en el espacio público y político es la condición primordial para la inclusión ciudadana, para la posesión plena de derechos de ciudadanía.

Por esta razón, como señalan A. Phillips (1995) y G. Procacci (1999), la ciudadanía es siempre un término político, en cuanto que supone la presencia y la representación simbólica de los grupos en la cosa pública y en las agendas políticas. De ahí que C. Tilly (1996: 1-17) establezca que la ciudadanía debe ser entendida en términos de exclusión e inclusión y como resultado de las luchas políticas entre colectivos e identidades, cuyos resultados dependen, en gran medida, del reconocimiento de grupo en la arena pública y política.

La literatura producida desde la ciudadanía diferenciada incorporará este tipo de consideraciones en su análisis, debido, por un lado, a las implicaciones que conllevan en la atribución y adquisición de derechos de ciudadanía para los niños y, por otro, a su utilidad para analizar la posición en la sociedad de estos como grupo diferente (la minoría de edad se asocia a unas características y a unas capacidades propias) a los adultos, en tanto que ostentan una ciudadanía de segunda. Según los autores, la definición de ciudadanía liberal predominante en las sociedades democráticas occidentales desplaza, necesariamente, a los niños a una condición de semiciudadanía (Cohen, 2005: 229), con estatus parcial (Roche, 1999: 482), ya que estos no son poseedores plenos de los derechos de ciudadanía, lo cual restringe principalmente sus derechos políticos y civiles.

Gran parte de la literatura plantea que la razón de dicha semiciudadanía es la persistencia de representaciones sociales e institucionales sobre la infancia y la adolescencia desde las cuales los niños y niñas son construidos en términos de diferencia en relación con los adultos en base a su inmadurez, su dependencia, su irracionalidad y su falta de autonomía. En consecuencia, J. Roche (1999: 485-486) plantea que, desde esta definición formal y legal de ciudadanía, los niños nunca podrán ser considerados ciudadanos plenos y deberán ser confiados a otros que representen sus intereses y velen por sus derechos en la sociedad. 
De acuerdo con estas afirmaciones, el enfoque de la ciudadanía diferenciada centra su interés en los nuevos significados de ciudadanía sustantiva que surgieron a raíz de las propuestas teóricas de los modelos de la diferencia, pues, para esta, resulta central la deconstrucción de los discursos, de las percepciones y de las actitudes adultas hacia la infancia, que se expresan en relaciones desiguales de poder y en las significaciones institucionales y normativas de lo que es «ser niño», por lo que busca transformar la noción tradicional de la ciudadanía para hacerla inclusiva a los niños (Cockburn, 1998: 112).

Como punto esencial desde el cual articulan toda una teoría sobre la ciudadanía de los niños, encontramos que algunos autores incorporan, a su análisis, la dialéctica entre libertad/igualdad y público/privado, presente en las teorías de la diferencia y en respuesta al modelo liberal de ciudadanía. En concreto, M. Moosa-Mitha (2005: 386) plantea la necesidad de incorporar como ciudadanos a los niños a través del reconocimiento público de sus diferencias — respecto de los adultos_-, teniendo en cuenta sus intereses y sus necesidades particulares como grupo (la minoría de edad). Esto es, que los niños y adolescentes tengan presencia política por sí mismos y no, exclusivamente, mediante la representación de la familia, del Estado o a través del principio del «mejor interés», pues, como establece E. F. Cohen (2005), en tanto que los niños no sean incorporados políticamente y no obtengan presencia pública como grupo, se perpetuará su condición de marginación respecto del espacio cívico y, en consecuencia, su estatus de semiciudadanía o ciudadanía delegada.

Así, B. Neale definió la ciudadanía para los niños como «un derecho al reconocimiento, al respeto y a la participación» (2004: 1). De hecho, para el común de la literatura producida en torno a la ciudadanía de la infancia, existe una falta de reconocimiento y de respeto hacia la contribución de los niños y adolescentes a la sociedad, a las comunidades y a las familias, lo cual imposibilita que estos sean considerados como ciudadanos plenos en el presente. Al respecto, el hecho de que los niños sigan, ideológicamente, representados como parte de la familia hace que sean especialmente invisibles en las cuestiones públicas como sujetos con intereses particulares. En este sentido, las medidas de protección significan, de alguna manera, la no participación y, en el fondo, mayor riesgo de vulneración de los derechos — de ciudadanía- de los niños. Según E. F. Cohen (2005: 229), esta privatización de la ciudadanía comporta serios riesgos de vulneración de los derechos niños y niñas pues, por un lado, las familias no pueden representar los derechos civiles y políticos de sus hijos $y$, por otro, el control, en términos de derechos, sobre la infancia puede ser usado en contra de la misma cuando, ante ella, prevalecen los intereses y los derechos de las familias.

Presencia y agencia son el reconocimiento («ser escuchados») de los niños en sus relaciones, en los espacios sociales de los que forman parte. De hecho, algunos autores insisten en que estos no llegan a ser ciudadanos en la práctica porque existen amplias barreras formales, estructurales y culturales que impiden su participación o una visión adultista de la misma. Como resultado de las asunciones institucionales y sociales sobre la infancia y la adolescencia, los 
niños son representados en términos de sujetos pasivos —a través de las ideas de dependencia, necesidad e irracionalidad-, lo cual les separa de la definición liberal de ciudadanos independientes y autónomos (Cohen, 2005: 236; James y James, 2004: 30-31; Lister, 2003: 101).

Por ello, según la literatura, la inclusión ciudadana de los niños requiere, por un lado, del énfasis en la participación y, por otro, de la reconsideración de la ciudadanía en un sentido sustantivo - más allá de un mero estatus de derechos-y como un proceso continuo de aprendizaje en la vida de las personas, en el cual se desarrollan prácticas de participación en las comunidades. Así mismo, para M. Moosa-Mitha (2005: 376), es necesario reconocer la posibilidad y la existencia de distintas formas de participación, significándolas dentro de las relaciones de interdependencia, más que en términos de autonomía.

En este sentido, los niños son diferentes, pero no «menos que» los adultos, y desarrollan también estrategias de interacción con los otros, expresan intereses, necesidades y desacuerdos o satisfacciones. Por lo que, para este enfoque, la participación de los niños debe considerarse en un sentido amplio, como la expresión de la capacidad para interaccionar en los múltiples espacios sociales de los que forman parte los niños, lo cual trasciende la dicotomía formada por lo público y lo privado ${ }^{16}$ (Cockburn, 2007; Plummer, 1999). Esta sería la vía para la inclusión ciudadana de los niños y la condición para su reconocimiento y su presencia pública como parte de la sociedad. Así, los derechos de los niños podrían dirigirse hacia el derecho de ser «diferentemente iguales» (Moosa-Mitha, 2005: 386). Por tanto, nos encontramos ante una definición de ciudadanía dinámica como un proceso que se construye y se transforma a través de prácticas.

En la línea de esta argumentación, cabe añadir que los niños — sobre todo aquellos que se acercan a la mayoría de edad- se hallan en una etapa de múltiples transiciones e inmersos en un proceso de desarrollo y de adquisición de derechos, obligaciones y responsabilidades asociadas con la ciudadanía (Lister, 2003: 101). Se trata de un periodo fundamental — de desarrollo y aprendizaje- en la formación de la identidad ciudadana y de sujetos autónomos.

En consecuencia, para este enfoque, se hace necesario abordar la ciudadanía esencialmente como prácticas de integración y de participación, donde es crucial la interrelación entre los sujetos de ciudadanía y las instituciones, para así entender qué procesos y qué estrategias interfieren o facilitan el acceso a la ciudadanía real y sustantiva de los niños. Siguiendo esta argumentación, el centro del debate en torno a la cuestión ciudadana en la infancia debería situarse en los procesos de adquisición de ciudadanía por su parte (apropiación de recursos de ciudadanía, competencias y desarrollo de sentimientos de pertenencia en las comunidades sociopolíticas e instituciones) y no en el carácter delegado de la ciudadanía y la situación de dependencia y tutela de los «menores».

16. En la teoría liberal, la esfera de lo privado, al que pertenecen, por ejemplo, el mundo de los cuidados y el ámbito familiar, se encuentra despolitizado. Cuestión que fue desafiada por la crítica feminista, al poner de manifiesto que dicho mundo de lo privado contiene relaciones de poder profundamente políticas. 


\subsubsection{De la agencia al reconocimiento (Lived citizenship)}

En la línea de pensamiento anteriormente presentada por la ciudadanía diferenciada, surge, como crítica y complementariedad, el enfoque de la ciudadanía vivida, partiendo de la consideración de que la ciudadanía, más allá de un estatus legal, es una práctica sociopolítica.

Este enfoque encuentra sus raíces en la corriente construccionista de la sociología de la infancia, por lo que definirá la cuestión de la ciudadanía de los niños y adolescentes como una construcción discursiva, creada, desarrollada y reelaborada por los sujetos sociales que participan en ella en contextos específicos, tanto "desde arriba» como «desde abajo». Es decir, la noción de ciudadanía vivida apela a los procesos - discursivos - a partir de los cuales los adultos atribuyen significado a la agencia de los niños (a lo largo de las diferentes esferas de sus vidas, por lo que la participación es facilitada o no) y a los procesos e interrelaciones a través de los cuales ellos interpretan y responden a dichas atribuciones.

De esta manera, para dicho enfoque, no resulta tan necesaria la revisión ni la transformación de la noción de ciudadanía — para hacerla más inclusivacomo el desvelar los discursos culturales a través de los cuales los niños son construidos como tales a partir de las políticas culturales de la infancia (James, 2011: 169).

Por tanto, para dicho enfoque, la noción de la ciudadanía de los niños se expresa en los «espacios» y en el «tiempo», por lo que existen diferentes concepciones de la misma, reflejo, a su vez, de las diferentes políticas culturales de la infancia, en las cuales las ideas culturales de los niños como personas del presente (being) o del futuro (becoming) desempeñan un rol central (James, 2011). Es a partir de las diferencias conceptuales en la identidad entre niños y adultos (Jenks, 1996) que surge el problema mismo de la situación de los niños como ciudadanos (James, 2011: 161).

No obstante, para entender la naturaleza de la ciudadanía de los niños y de los adolescentes, es necesario explorar y prestar atención, en contextos particulares, no solo al modo cómo la ciudadanía legal se hace asequible para los niños, sino también (y sobre todo) al modo como esta responde ante dicha idea a través de sus experiencias y del ejercicio de su agencia en sus vidas cotidianas (James, 2011: 173). El desplazamiento del análisis institucional hacia las experiencias vitales de los niños, respecto a este, retoma la noción de reproducción interpretativa (Corsaro, 1997: 18-19), para poner de manifiesto que los niños no se limitan meramente a interiorizar la sociedad y la cultura, sino que también contribuyen activamente a promoverlas y a cambiarlas.

Es decir, desde este enfoque, la ciudadanía, los discursos sobre ella y las estructuras formales que la facilitan, o no, no son considerados un marco estático y determinante, sino que más bien los sujetos sociales (niños y adolescentes) transforman ese marco, apropiándose de él y pujando por el establecimiento de nuevos significados.

En consecuencia, para el enfoque de la ciudadanía vivida, la ciudadanía es entendida, eminentemente, como un proceso relacional construido, además, 
«desde abajo», pues la implicación y la participación activa de los niños, según M. Liebel (2008), no se limita a la participación institucionalizada en la cual los adultos ofrecen la posibilidad a los niños de «formar parte de» la toma de decisiones, en un sentido estrictamente democrático y organizado, sino que, más allá de ello, implica una consideración real de las posibilidades de los niños desde la práctica en su vida cotidiana, teniendo en cuenta que son personas y ciudadanos del presente.

La clave de este enfoque se va dirigiendo, por tanto, hacia el propio proceso de construcción de la ciudadanía desde abajo (citizenship from below), expresada por los propios niños, para poder hablar de su ciudadanía en un sentido pleno (Liebel, 2008: 42).

Teniendo en cuenta que la ciudadanía es un proceso relacional y construido, también, desde abajo, se hace especialmente relevante el analizar cómo los niños experimentan y responden a la imagen adulta de lo que es la infancia y ser niño, estableciendo el énfasis de los análisis en la idea de agencia. Se trata de conocer cómo los niños ejercen sus derechos en su vida cotidiana (James, 2011: 172).

En este sentido, frente a la propuesta de la ciudadanía de los niños como una ciudadanía basada en la igualdad diferenciada, el enfoque de la ciudadanía vivida propone repensar e incorporar a los niños y adolescentes como ciudadanos a través de su agencia, ya sea desde las contribuciones de estos a la sociedad en el ejercicio de lo cotidiano o desde los movimientos sociales protagonizados por los propios niños a nivel internacional (Liebel, 2008), pues el problema de la consideración de la ciudadanía de los niños desde la perspectiva de la diferencia es que la ciudadanía sobre esta base (igualdad diferenciada) solo puede tener un tamaño infantil (Jans, 2004: 38).

\section{Implicaciones de los debates sobre la ciudadanía e infancia}

En mayor o menor medida, el corpus teórico de los debates contemporáneos sobre la ciudadanía de los niños y adolescentes cuestiona la idea del menor desarrollada por las disciplinas psicológicas y pedagógicas ${ }^{17}$. Así, desde las distintas corrientes y los enfoques llevados a cabo, existe un intento profundo en reconceptualizar la imagen social y colectiva de la infancia, de sus capacidades y aptitudes para la participación social y política.

En primer lugar, la literatura producida en torno esta cuestión coincide en presentar al niño como un actor social y a definir la ciudadanía en términos procesuales. Es decir, "la última condición moderna permite presentar a los niños, crecientemente, como actores sociales por sí mismos, dentro y fuera de la familia. La ciudadanía de los niños está basada en un proceso de aprendizaje continuo, en el cual niños y adultos son interdependientes» (Jans, 2004: 40).

17. Referidas a las representaciones de la infancia en torno al paradigma de la edad, en clave de dependencia e inmadurez, que tanto cuestionó la sociología de la infancia. 
Ahora bien, la diferencia fundamental es que, para la corriente legal - exceptuando el enfoque de Child Liberationist-, el menor sigue siendo, en su esencia, un ciudadano del futuro, mientras que la corriente sustantiva lo propone desde y en el presente. Dentro de la primera, el enfoque proteccionista, animado por lo establecido en la CDN de 1989, sostiene que la participación de los niños en la sociedad, así como en los procesos de toma de decisiones, ha de ser promovida y facilitada, en general, desde el mundo adulto. En este sentido, la escuela o los gobiernos locales ocupan un lugar fundamental. La mayor parte de la literatura sobre ciudadanía e infancia se engloba en esta postura y produce numerosos trabajos e investigaciones en torno a tres líneas temáticas fundamentales:

1. Las percepciones de los niños en torno a la ciudadanía y a la nacionalidad (Hine, 2004; Lister, 2003; Lister et al., 2003; Taylor y Smith, 2009).

2. La promoción y el conocimiento, por parte de los niños, de sus derechos y responsabilidades (Melton y Limber, 1992).

3. La contribución infantil y juvenil en el desarrollo sostenible (Chawla y Heft, 2002).

Es así como, en los últimos veinte años, gran parte de las estrategias internacionales, guiadas por lo establecido en la CDN de 1989, ha establecido su epicentro en la idea de participación (democrática) de los niños y adolescentes en el mundo que les rodea (Kjørholt, 2002: 75).

No obstante, como sugieren D. Bühler-Niederberger y R. Van Krieken (2008: 148), la dificultad de los discursos normativos que establecen el énfasis en la agencia de los niños, así como en la importancia del reconocimiento de su voz, es que, en primer lugar, no terminan de incorporar las diferencias ni las distinciones entre los niños, especialmente las que se relacionan con las desigualdades sociales y de clase.

Estas desigualdades y diferencias han sido, precisamente, decisivas en la emergencia histórica de la idea "moderna de infancia», así como en la formación del imaginario político y profesional en torno a la misma. Por tanto, para los autores, la idea de la agencia del niño no debe ser interpretada, meramente, como que él es por sí mismo un actor social más allá de su estatus y clase, como un actor puramente individual. Más que eso, los niños y niñas actúan en el marco de estructuras sociales, económicas y políticas, que significan y, también a veces, limitan sus posibilidades de acción.

La infancia contemporánea, pues, continúa posicionada entre la concepción moderna de los derechos del niño (CDN de 1989) y las prácticas y las rutinas que producen y preservan las desigualdades sociales. De ahí la importancia de los planteamientos iniciados por la corriente sustantiva, los cuales permiten trasladar a la discusión dominante los elementos que interfieren y/o que significan la ciudadanía de los niños y/o adolescentes. 


\section{Referencias bibliográficas}

Archard, D. (2004). Children, Rights and Childhood. Londres: Routledge.

ArIÈs, P. (1962). Centuries of Childhood. Londres: Jonathan Cape.

Barbalet, J. M. (1988). Citizenship: Rights, struggle and class inequality. Milton Keynes: Open University Press.

Ben-Arieh, A. y Boyer, Y. (2005). «Citizenship and Childhood: The State of Affaire in Israel». Childhood [en línea], 12 (1), 33-53.

<http://dx.doi.org/10.1177/0907568205049891>.

Benedicto, J. (2007). Infancia y juventud. Nuevos sujetos de ciudadanía: Un reto para la sociología de la infancia. Trabajo presentado en El Espacio Social de la Infancia. San Martín de Valdeiglesias. Curso Internacional de Verano.

Benedicto, J. y Morán, M. L. (2002). La construcción de una ciudadanía activa entre los jóvenes. Madrid: INJUVE.

BüHler-Niederberger, D. y Van Krieken, R. (2008). «Persisting Inequalities: Childhood between global influences and local traditions». Childhood [en línea], 15 (2), 147-155. <http://dx.doi.org/10.1177/0907568207088419>.

Burtt, S. (2003). "The Proper Scope of Parental Authority». En: Macedo, S. y Young, I. M. (eds.). Child, Family and State. Nomos XLIV. Nueva York: University Press.

Chawla, L. y Heft, H. (2002). "Children's competence and the ecology of communities: A functional approach to the evaluation of participation». Journal of Environmental Psychology [en línea], 22, 201-216. <http://dx.doi.org/10.1006/jevp.2002.0244>.

Cockburn, T. (1998). "Children and Citizenship in Britain: A Case for a Socially Interdependent Model of Citizenship». Childhood [en línea], 5 (1), 99-117. <http://dx.doi.org/10.1177/0907568298005001007>.

- (2007). «Reconstructing Children's Agency: Boundaries of Rights and Care». En: Beckett, C.; Heathcote, O. y Macey, M. (eds.). Negotiating Boundaries?: Identities, Sexualities and Diversities. Cambridge: Cambridge Scholars Publishing.

Cohen, E. F. (2005). «Neither Seen Nor Heard: Children's Citizenship in Contemporary Democracies». Citizenship Studies [en línea], 9 (2), 221-240. <http://dx.doi.org/10.1080/13621020500069687>.

Cohen, H. (1980). Equal Rights for Children. Totowa y Nueva York: Littlefield, Adams and Company.

Corsaro, W. (1997). The Sociology of Childhood. Thousand Oaks, CA: Pine Forge.

EArLs, F. (2011). "Children: From Rights to Citizenship». The ANNALS of the American Academy of Political and Social Science [en línea], 633 (1), 6-16. <http://dx.doi.org/10.1177/0002716210383637>.

FARsOn, R. (1974). Birthrights. Londres: Collier Macmillan.

Faulks, F. (2003). Citizenship. London: Routledge.

Fineman, M. (2003). "Taking Children's Interest Seriously». En: Macedo, S. y Young, I. M. (eds.). Child, Family and State, Nomos XLIV. Nueva York: University Press.

Francisco, A. de (2007). Ciudadanía y democracia: Un enfoque republicano. Madrid: Catarata.

Franklin, B. (1986a). The Rights of Children. Oxford: Basil Blackwell.

- (1986b). «Introduction». En: Franklin, B. (ed.). The Rights of Children. Oxford: Basil Blackwell. 
Freeman, M. (1992a). «The Limits of Children's Rights». En: Veerman, P. (ed.). The Ideologies of Children's Rights. Dordrecht: Martinus Nijhoff.

Galston, W. (2003). «Parents, government and children». En: Macedo, S. y Young, I. M. (eds.). Child, family and state: Nomos XLIV. Nueva York: New York University Press.

Goldstein, J.; Freud, A. y Solnit, A. J. (1979). Beyond the Best Interest of the Child. Londres: Brunett Books.

Hackett, C. (1997). «Young People and Political Participation». En: Roche, J. (ed.). Youth in Society. Londres: Sage.

Hill, M. y Tisdall, K. (1997). Children and Society. Harlow: Prentice Hall.

Hine, J. (2004). Children and citizenship [en línea]. Londres: Home Office Online Report 08/04. <http://dera.ioe.ac.uk/8474/2/rdsolr0804.pdf>.

Holt, J. (1975). Escape from Childhood. Harmondsworth: Penguin.

Hougalte, L. (1980). The Child and the State: A Normative Theory of Juvenile Rights. Baltimore y Londres: Johns Hopkins University Press.

James, A. (2011). "To be (come) or not to be (come): Understanding children's citizenship». The ANNALS of the American Academy of Political and Social Science [en línea], 633 (1), 167-179. <http://dx.doi.org/10.1177/0002716210383642>.

James, A.; Curtis, P. y Birch, J. (2008). "Care and Control in the Construction of Children's Citizenship». En: Invernizzi, A. y Williams, J. (eds.). Children and Citizenship. Londres: Sage.

James, A. y James, L. A. (2004). Constructing Childhood: Theory, policy and social practise. Basingstoke: Palgrave Macmillan.

Jans, M. (2004). «Children as Citizens: Towards a Contemporary Notion of Children Participation». Childhood [en línea], 11 (1), 27-44. <http://dx.doi.org/10.1177/0907568204040182>.

Jenks, C. (1996). Childhood. Londres: Routledge.

Jones, G. y Wallace, C. (1992). Youth, Family and Citizenship. Buckingham: Open University Press.

KING, M. (2007). "The Sociology of Childhood as a Scientific Communication». Childhood [en línea], 14 (2), 193-211. <http://dx.doi.org/10.1177/0907568207078327>.

Kirbi, P. y Woodhead, M. (2003). "Children's Participation in Society». En: MonTgomery, H. (ed.). Changing Childhood: Social and Global. Nueva York: Open University.

Kјørнolt, A. T. (2002). "Small is Powerful: Discourses on "Children and Participation" in Norway». Childhood [en línea], 9 (1), 63-82. <http://dx.doi.org/10.1177/0907568202009001005>.

Knutsson, K. E. (1997). Children: Noble Causes or Worthy Citizens? Florencia: Arena; UNICEF.

Lansdown, G. (2003). «Reading: The Participation of Children». En: Montgomery, H. (ed.). Changing Childhood: Social and Global. Nueva York: Open University Press.

LeE, N. (2005). Childhood and Society: Growing up in an age of uncertainly. Londres: Open University Press.

Liebel, M. (2006a). «Los movimientos de los niños y niñas trabajadores: Un enfoque desde la sociología». Política y Sociedad, 43 (1), 105-123.

- (2006b). «Entre protección y emancipación: Derechos de la infancia y políticas sociales». Experto en Políticas Sociales de la Infancia de la UCM [en línea], 1. <http://www.ucm.es/info/polinfan/2007/Serie_teorica_1_UCM.pdf>. 
- (2008). "Citizenship from below: Children's rights and social movements». En: Williams, J. y Invernizzi, A. (eds.). Children and Citizenship. Londres: Sage.

Lister, R. (2003). "Acquiring Responsibilities and Citizenship: Social Participation and Social Responsibilities». En: Chisholm, L. (ed.). Family forms and the young generation in Europe. Viena: Osterreichisches Institut fur Familienforschung.

Lister, R.; Smith, N.; Middleton, S. y Cox, L. (2003). «Young People Talk about Citizenship: Empirical Perspectives on Theoretical and Political Debates». Citizenship Studies [en línea], 7 (2), 235-253.

<http://dx.doi.org/10.1080/1362102032000065991>.

Mandel, R. (1995). "Second-generation Noncitizens: Children of the Turkish Migrant Diaspora in Germany». En: Stephens, S. (ed.). Children and the politics of culture. Princeton: Princeton University Press.

Marshall, T. H. (1998). Ciudadanía y clase social. Madrid: Alianza.

Melton, G. B. y Limber, S. P. (1992). «What rights mean to children: Cross-cultural perspectives». En: Veerman, P. (ed.). Ideologies of children's rights. Dordrecht: Martinus Nijhoff.

Moosa-Mitha, M. (2005). "A Difference-Centred Alternative to Theorizing of Children's Rights». Citizenship Studies [en línea], 9 (4), 369-388. $<$ http://dx.doi.org/10.1080/13621020500211354>.

Neale, B. (2002). "Dialogues with Children: Children Divorce and Citizenship». Childhood [en línea], 9 (4), 455-475. <http://dx.doi.org/10.1177/0907568202009004006>.

O’NeIll, O. (1992). "Children's Right and Children's Lives». International Journal Law Policy Family, 6 (1), 24- 42.

Phillips, A. (1995). The Politics of Presence. Oxford: Clarendon Press.

Plummer, K. (1999). Inventing intimate citizenship. Trabajo presentado en Rethinking Citizenship Conference. University of Leeds.

Procacci, G. (1999). "Ciudadanos pobres, la ciudadanía social y la crisis de los Estados del Bienestar». En: Luckes, S. (ed.). Ciudadanía: Justicia social, identidady participación. Madrid: Siglo XXI.

Rawls, J. (1971). A theory of Justice. Cambridge MA: Harvard University Press.

Roberts, M. (2001). "Childcare Policy». En: Foley, P.; Roche, J. y Tucker, S. (eds.). Children in Society: Contemporary Theory, Policy and Practice. Hampshire y Nueva York: Palgrave.

Roche, J. (1999). "Children: Rights, Participation and Citizenship». Childhood [en línea], 6 (4), 475-493. <http://dx.doi.org/10.1177/0907568299006004006>.

SASSEN, S. (ed.) (2003). Contrageografias de la globalización: Género y ciudadanía en los circuitos transfronterizos. Madrid: Traficantes de Sueños.

Shachar, A. (2003). «Children of Lesser State: Sustaining Global Inequality through Citizenship Law». En: Macedo, S. y Young, I. M. (eds.). Child, Family and State. Nomos XLIV. Nueva York: University Press.

Shamgar-Handelman, L. (1994). «To Whom Does Childhood Belong?». En: QvorTrup, J. (ed.). Childhood Matters: Social Theory, Practice and Politics. Aldershot: Avebury.

Sirm, B. (2000). Gender and Citizenship: Politics and Agency in France, Britain and Denmark. Cambridge: Cambridge University Press.

Stasiulis, D. (2002). "The Active Child Citizen: Lesson form Canadian Policy and the Children's Movement». Citizenship Studies, 6 (4), 507-538. 
Such, E. y Walker, R. (2005). «Young citizens or Policy Objects?: Children in the "Rights and responsibilities" debates». Journal of Social Policy [en línea], 34 (1), $39-57$. <http://dx.doi.org/10.1017/s0047279404008256>.

TAYlor, N. y Smith, A. B. (eds.) (2009). Children as citizens: International voices. Dunedin, N. Z.: Otago University Press.

Thomson, R.; Holland, R. McGrellis, S.; Bell, R.; Henderson, S. y Sharpe, S. (2004). «Inventing Adulthoods: A Biographical Approach to Understanding Youth Citizenship». Sociological Review [en línea], 52 (2), 139-294. <http://dx.doi.org/10.1111/j.1467-954x.2004.00466.x>.

Tilly, C. (1996). «Citizenship Identity and Social History». En: Tilly, C. (ed.). Citizenship, identity and social history. Cambridge: Cambridge University Press..

Turner, B. (1993). Citizenship and Social Theory. Londres: Sage.

VAn Bueren, G. (2011). «Multigenerational Citizenship: The Importance of Recognizing Children as National and International Citizens». The ANNALS of the American Academy of Political and Social Science [en línea], 633 (1), 30-51. $<$ http://dx.doi.org/10.1177/0002716210383113>.

Wintersberger, H. (2006). "Childhood and Citizenship: The Generational Order of the Welfare State». Politica y Sociedad, 43 (1), 81-103.

Young, I. M. (2000). La justicia y la política de la diferencia. Madrid: Cátedra. 